\section{The future is getting closer: machine reading of primary-care clinical records to predict future diagnoses}

\section{Imogen Schofield ${ }^{1}$, Noel Kennedy ${ }^{1}$, Dave Brodbelt ${ }^{1}$, Stijn Niessen ${ }^{1,2}$, David Church ${ }^{1}$, Rebecca Geddes ${ }^{1}$, Dan O'Neill ${ }^{1}$}

1 Royal Veterinary College, Hatfield, United Kingdom

2 The VetCT Telemedicine Hospital, Cambridge, United Kingdom

\section{OBJECTIVES}

To apply artificial intelligence (Al) methods to primarycare veterinary electronic patient records (EPRs), to predict future clinical diagnoses in veterinary practice.

\section{METHODS}

The VetCompass ${ }^{\mathrm{TM}}$ programme shares anonymised EPRs (clinical notes, demography and treatment data) with UK primary-care veterinary practices. To harness the full power of Big Data, Al methods are required. A pilot study on one condition, Cushing's syndrome (CS), applied natural language processing (NLP) to a sample of VetCompass ${ }^{T M}$ EPRs. A long short-term memory (LSTM) deep learning algorithm was trained to predict future diagnoses by examining patients' clinical notes recorded up to 30 days prior to the first mention of CS. The model was validated using historical records of dogs with and without CS.

\section{RESULTS}

VetCompass ${ }^{\mathrm{TM}}$ covers 1,803 UK veterinary clinics, 10 million animals and 55 million clinical notes. From a sample of 905,544 dogs, 10,141 dogs with a suspicion of Cushing's syndrome were identified. Following NLP-analysis of clinical notes, the LSTM algorithm classified these dogs as future diagnoses ( $n=1,066 ; 10.5 \%$ ) and nondiagnoses $(9,075 ; 89.5 \%)$ of CS. The developed algorithm showed good discrimination with an AUROC $=0.74(95 \%$ $\mathrm{Cl} 0.73-0.74)$.

\section{STATEMENT (CONCLUSIONS)}

This pilot study demonstrates the applicability of $\mathrm{Al}$ methodology to facilitate diagnosis of disease earlier in the clinical trajectory of sub-acute disorders such as CS. Earlier diagnosis should improve both animal welfare and clinical outcomes. 